\title{
De Chronos à Kairos : la communication dans l'urgence existentielle
}

Nicole Giroux et Anne Boucher

\section{OpenEdition}

1 Journals

Édition électronique

URL : http://journals.openedition.org/communicationorganisation/3394

DOI : 10.4000/communicationorganisation.3394

ISSN : 1775-3546

Éditeur

Presses universitaires de Bordeaux

\section{Édition imprimée}

Date de publication : 1 juin 2006

Pagination : 140-162

ISSN : 1168-5549

\section{Référence électronique}

Nicole Giroux et Anne Boucher, « De Chronos à Kairos : la communication dans l'urgence

existentielle ", Communication et organisation [En ligne], 29 | 2006, mis en ligne le 21 juin 2012, consulté le 18 décembre 2020. URL : http://journals.openedition.org/communicationorganisation/3394 ; DOI : https://doi.org/10.4000/communicationorganisation.3394

Ce document a été généré automatiquement le 18 décembre 2020.

(C) Presses universitaires de Bordeaux 


\title{
De Chronos à Kairos : la communication dans l'urgence existentielle
}

\author{
Nicole Giroux et Anne Boucher
}

"L'urgence, on le sait, a à voir avec la mort ". Michel De M'Uzan, Aux confins de l'identité,

Paris : Gallimard, 2005 : 92

1 Il existe plusieurs figures de l'urgence, tant dans les situations vécues que dans les représentations qui en sont faites. Nous voulons réfléchir ici sur la notion d'urgence existentielle vécue par la personne au stade palliatif d'une maladie fatale. Nous voulons aborder cette question délicate à partir du point de vue des personnes qui acceptent de l'accompagner dans ce dernier périple. Cette urgence existentielle, parce qu'elle est le lot de la majorité des humains ${ }^{1}$, nous est apparue offrir une opportunité de comprendre ce phénomène de la mort, phénomène qui a la particularité d'être à la fois, unique, prévisible et général. Ce phénomène nous le concevons à la fois comme un fait objectif, mais aussi comme un processus, une expérience qui est vécue, interprétée et racontée ; en somme, comme une réalité subjective. Cette réalité subjective gagnerait, croyonsnous, à être mieux connue car c'est une « urgence annoncée » à laquelle nous serons pour la plupart éventuellement confrontés. Cette meilleure connaissance pourrait bien nous aider alors à assumer notre finitude, « à avoir accès à une relation plus détendue avec l'inéluctable $»^{2}$

2 Dans ce texte, nous définirons le concept d'urgence employé dans cette recherche empirique. Puis, nous présenterons la problématique à l'origine de cette étude. Par la suite nous décrirons l'organisation observée, l'échantillon ${ }^{3}$ et la méthodologie choisie. Les résultats des entrevues réalisées seront rapportés à partir de l'analyse thématique qui en a été faite. Dans la discussion qui suivra, les notions d'urgence et de communication seront reprises pour les mettre en perspective. 


\section{Qu'est-ce que l'urgence?}

3 Dans notre réflexion, la définition de l'urgence retenue est la suivante : le concept d'urgence fait référence à une temporalité limitée, à un enjeu capital qui suscite le désir d'intervenir pour améliorer la situation ou pour éviter des conséquences négatives. En somme, on parle d'urgence quand le temps presse. Il presse quand on veut gagner du temps, quand le temps est compté ou quand on n'a plus le temps.

Pour comprendre ce phénomène, nous en avons retenu deux dimensions : 1) la source, soit l'urgence endogène et exogène ; 2) la cible, soit l'urgence individuelle et collective. Ces dimensions combinées, on constate qu'il y a l'urgence que l'individu se crée luimême pour mettre de l'adrénaline et de l'intensité dans sa vie. Il y a aussi la pression sur le temps par laquelle les dirigeants de l'organisation veulent motiver leurs employés à produire davantage. Ce sont là deux exemples de l'urgence artificielle.

Il y a l'urgence provoquée par des accidents technologiques (Tchernobyl, Bhopal) ou par des catastrophes naturelles (ouragan, tremblement de terre, tsunami) qui touchent à la fois les individus et les organisations. Ce sont des exemples de l'urgence événementielle.

6 Il y a enfin une troisième forme d'urgence qui touche chaque individu et toute la collectivité humaine, c'est l'urgence existentielle, l'urgence prévisible et annoncée de nos derniers moments.

7 C'est une urgence parce que :

- pour le patient, le temps est désormais compté ;

- l'enjeu pour lui est important : il est rendu à l'étape du bilan de vie où, par la réminiscence, il tente de donner sens à sa vie, de faire le deuil de son identité sociale, de régler « ses affaires non terminées » et, comme le dit Michel de M'Uzan, de se mettre au monde avant d'en partir ${ }^{4}$;

- quand le temps est compté, les interventions les plus significatives sont sans doute celles qui permettent justement la création de signification : ce sont les actes de communication.

Il faut se rappeler que le terme communication (du latin cum unicare) réfère à la fois à un processus et à un contenu. Il décrit à la fois l'acte de «s'unir avec » pour entrer en relation, créer des liens et celui de "mettre en commun ", partager des idées, des opinions, des affects. Le besoin de communiquer est particulièrement fort durant les derniers moments de vie et plusieurs malades craignent moins la mort elle-même que de devoir l'affronter seul. Paradoxalement, dans ce processus progressif de détachement, de lâcher prise, la présence de l'autre apparaît cruciale et le lien humain est ardemment désiré.

L'autre est recherché car il est là :

- pour provoquer, c'est-à-dire appeler, la parole par l'écoute attentive (Feldman-

Desrousseaux, 2001 : 455 );

- pour recevoir ce que le malade a besoin de dire; pour lui permettre de se dire à lui-même des choses qui le feront grandir dans sa propre vérité (Feldman-Desrousseaux, 2001 : 456) ;

- pour l'aider à raconter ce qu'il est en train de vivre et ainsi donner sens à cette expérience nouvelle de la proximité de la mort, ce qui peut l'aider à traiter toutes ces informations nouvelles et à s'acclimater à une situation perturbante (Skott, 2001) ;

- pour offrir « un espace symbolique de liberté de confidentialité où tous les sentiments qui déferlent dans la fin de vie peuvent être exprimés » (Kebers, 2005 :185) ; 
- pour créer progressivement une relation de confiance permettant à la personne d'exprimer ses besoins physiques, psychologiques et spirituels (Raudonis, 1995);

- pour promouvoir, par sa présence rassurante, un sentiment de sécurité chez le patient ainsi qu'une impression de contrôle sur sa propre vie jusqu'à la fin (Appelin et al., 2005) ;

- pour témoigner, par des visites régulières, des marques d'attention et une écoute respectueuse, l'intérêt qu'il accorde à cet individu; pour lui montrer qu'il le considère comme un sujet, un humain encore vivant, un être de valeur (Foster, 2002) ;

- pour le suivre et le soutenir, le cas échéant, dans son périple de transformation qui aboutit selon le modèle de Kübler-Ross (1969), à la résignation ou à l'acceptation après avoir fait le deuil de ses multiples pertes : perte de la maîtrise de son corps, perte de son identité sociale, de ses relations (Sourkès, 1982 );

- pour éventuellement l'aider à parvenir à « ce laisser-être qui rend toutes choses à ellesmêmes, du moment que l'on cesse de les asservir à nos projets et que l'on parvient à se dépouiller de soi »(Dastur, $2005: 84)$;

- pour permettre à l'accompagné de mener son existence à terme (Hirsch, $2004: 166)$ « de pouvoir partir en ayant malgré tout le sentiment ou la sensation d'un achèvement dont la mémoire sera préservée »;

- pour recevoir avec gratitude ce don de communication. Comme le décrit si bien de M'Uzan, le malade en fin de vie est un être en désir de relation. Il souhaite la venue d'un alter, d'un interlocuteur, d'une personne qui va accepter de l'accompagner dans ce dernier voyage. D'ailleurs, Suzanne Hervieux (2005: 85) rappelle que le terme accompagner, selon l'étymologie, signifie «partager le pain avec quelqu'un " et que dans le contexte des soins palliatifs, "ce pain est une souffrance immense et un travail psychique intense pour le malade qui doit quitter tous ses «lieux » et faire son «travail de trépas ». C'est une épreuve pour ses proches, délogés de leurs habitudes et dans les affres de la séparation.» Dans ce contexte, la présence d'une personne avec qui partager ces moments est appréciée puisque, comme le propose Marie de Hennezel (2005), «l'accompagnement permet de ne pas mourir avant le temps », il vient répondre à ce besoin ultime du mourant " d'être en relation " ${ }^{5}$ aussi longtemps qu'il le souhaite.

\section{La problématique de l'urgence existentielle accompagnée :}

\section{la « communication compatissante "}

12 Le passage du temps chronologique de la vie ordinaire aux «instants précieux » des derniers moments, le kairos ${ }^{6}$, est favorisé par l'existence "d'anges gardiens ». Ces personnes peuvent être les proches, les soignants, les aumôniers ou les bénévoles qui accompagnent le mourant en fin de vie.

13 Ces personnes agissent dans le cadre d'organisations médicales, paramédicales, d'associations caritatives qui doivent œuvrer de concert pour offrir des services au patient. Cela suppose une bonne concertation des parties et une bonne coordination des interventions. Des mécanismes et dispositifs de communication (ligne d'information, cahier de soins, table de concertation, etc.) sont alors mis en place pour assurer la coorientation des partenaires, la répartition des soins, le suivi des dossiers. Il s'agit de la « communication organisante ». 

communication compatissante. Cet accompagnement a été défini comme une « relation de réciprocité, une communication profonde et humaine entre deux personnes en des moments d'intense émotion de leur existence ${ }^{7}$. Quant à la compassion, elle est décrite par Kanov et al. (2004) comme une partie essentielle de l'existence humaine et de la vie en organisation. Ces auteurs la définissent comme une réponse empathique suscitée par la souffrance de l'autre qui stimule le désir d'agir pour la réduire ou la rendre plus tolérable. Les accompagnateurs peuvent ainsi, dans les moments difficiles, faciliter la prise de parole, l'expression des émotions, l'apaisement de la douleur physique et psychique, par leur présence réconfortante, leur écoute attentive, leur silence accueillant et leur toucher apaisant ${ }^{8}$. «bonne mort "9. Toutefois, accompagner ce n'est pas guider en fonction d'une représentation préétablie de la mort ${ }^{10}$. C'est plutôt suivre le malade dans son cheminement personnel, accepter qu'il vive ses derniers moments comme il le désire avec l'assurance d'être accepté, quoi qu'il arrive (de Montigny et de Hennezel, 1990).

on Néron (1995), il est alors primordial de s'adapter à la temporalité du malade qui tourne autour de deux points de repères : l'avenir du temps qui lui reste et la phase terminale qui est le temps de la séparation et du détachement. Il s'agit donc de l'accompagner en maintenant la distance qu'il souhaite entretenir avec la réalité de sa propre fin.

17 La littérature prescriptive tend à idéaliser ces moments de communication interpersonnelle. Elle tend à passer sous silence les exigences et les difficultés de la communication dans ce contexte particulier. Pour en savoir davantage sur la question, il nous a semblé pertinent d'approfondir cette question par une étude empirique.

L'objectif de la présente recherche est donc de décrire cette relation de communication à partir du vécu des bénévoles, ces intervenants dont la contribution est habituellement peu prise en compte dans les études sur le fonctionnement des organisations de santé. Pourtant, d'après Marie Sylvie Richard (2003 : 132-133) une spécialiste en soins palliatifs, leur présence est essentielle car ils participent « comme les soignants à la recréation du tissu social » et «contribuent à l'élaboration du sens ». S'appuyant sur les travaux de Matray ${ }^{11}$, elle justifie la présence du bénévolat en soins palliatifs par leurs rôles politique (affirmation de la signification de la fin de vie dans une société qui tend à l'occulter) et éthique (« assumer les risques de la présence auprès de l'autre, proche de la mort, dans un lien fondamental d'humanité»).

\section{Le contexte organisationnel}

Pour mieux comprendre la communication en situation d'urgence existentielle, nous avons interrogé des bénévoles visitant des patients cancéreux en phase terminale. Ces personnes sont encadrées au sein d'une association qui offre des services de soins palliatifs à domicile. Cette association, financée par des dons privés, fournit des services aux personnes atteintes de cancer en phase pré-terminale et terminale. Elle œuvre depuis déjà plusieurs décennies et offre différents services, le principal étant les visites à domicile d'infirmières diplômées qui fournissent aux patients les soins prescrits par les médecins traitants et qui assurent le suivi médical. Les patients sont 
référés par la famille, le médecin omnipraticien, l'oncologue du Centre hospitalier universitaire ( $\mathrm{CHU}$ ) ou encore la travailleuse sociale du centre local de service communautaire (CLSC) si celui-ci n'offre pas un tel service. L'Association offre des services d'accompagnement (transport et soutien) lors des visites à l'hôpital, l'accompagnement sous forme de répit à la famille (présence auprès du patient), de même que l'accompagnement psychologique et l'accompagnement spirituel.

Les bénévoles qui prennent en charge cet accompagnement sont recrutées par des annonces dans les journaux. Elles doivent passer une entrevue de sélection avec la responsable du recrutement et reçoivent une formation de plusieurs dizaines d'heures étalées sur plusieurs mois. Cette formation traite des aspects pratiques (les déplacements, quoi faire en cas d'urgence médicale), psychologiques (la juste distance, les attentes du patient, de la famille, de l'accompagnant) communicationnels (le secret professionnel, le respect des juridictions, la non intrusion, l'écoute) spirituels (le respects des valeurs et des pratiques de chacun). De plus, à tous les mois, les bénévoles qui souhaitent y participer, sont conviées à des sessions de ressourcement pour des mises à jour de la formation, des conférences et des échanges d'expériences entre accompagnatrices. Les bénévoles s'engagent à passer six heures par semaine auprès du malade. L'accompagnement se termine en principe ${ }^{12}$ lorsque le patient est hospitalisé durant les dernières semaines de sa maladie.

\section{La méthodologie}

21 Pour comprendre la relation de communication vécue par les bénévoles dans ce contexte d'urgence particulier, dix entrevues selon la tradition phénoménologique ont été faites. La phénoménologie a été privilégiée puisqu'elle semblait la plus adaptée à notre objet de recherche comme perspective et comme méthode. Comme le présente Boucher (2004), les phénoménologues ont montré que l'autre et le langage occupent une place centrale dans l'expérience humaine. Ainsi, pour Heidegger, être, c'est essentiellement être en compagnie de l'autre; pour Merleau-Ponty, l'autre participe activement à la constitution de l'expérience du sujet ; alors que pour Gadamer, c'est la communication qui permet la compréhension, cette rencontre entre l'humain et le monde; finalement, pour Ricoeur, la compréhension de soi est médiatisée par le langage symbolique. En résumé, elle affirme: «ces quatre philosophes...révèlent que l'expérience humaine est langagière, c'est-à-dire qu'elle s'exprime et se constitue dans la communication avec autrui » (Boucher, $2004: 57$ ).

22 Cette étude s'intéresse donc à l'interprétation que les bénévoles font de leur expérience de communication avec les patients en soins palliatifs à travers les narrations qu'ils en font. Il s'agit ici d'une approche à la fois narrative (Giroux et Marroquin, 2005) et herméneutique des témoignages des répondants (Boucher, 2004). Ces entrevues semidirigées, d'une durée variant de une à deux heures, ont été enregistrées puis transcrites dans leur intégralité. Ces données ont été traitées, dans le cadre du présent article, par l'analyse thématique.

23 L'échantillon de répondants était composé en majorité de femmes, seulement deux hommes ont accepté de participer à notre recherche. Cet échantillon correspond, selon la responsable des bénévoles, à la composition de la population des personnes faisant de l'accompagnement sous forme de répit. Les membres de l'échantillon sont surtout des femmes au début de la cinquantaine, jeunes retraitées ou femmes au foyer qui 
peuvent consacrer au moins six heures semaines à l'accompagnement. La plupart ont déjà connu la perte d'un proche avant de s'engager dans l'accompagnement. Les accompagnateurs masculins sont eux plus âgés (60-70 ans). Ils font surtout de l'accompagnement au transport et de l'accompagnement spirituel.

L'intensité de l'expérience et la qualité de la communication semblent être des raisons justifiant leur engagement. Spontanément, une répondante exprime sa motivation de la manière suivante : « j'accompagne les gens en fin de vie parce que je pense qu'ils sont encore vivants ». Une autre raconte «Pouvoir partager ces vies...Il a dit qu'il ne l'avait jamais vraiment montré à personne (une œuvre qu'il avait fait)...la plénitude...Ce petit moment là, de savoir que je suis à la bonne place au bon moment...il est décédé quelques jours après...on est des témoins. » Une troisième dit : « Les gens qui vont mourir, ils n'ont pas de masque. C'est comme communiquer de l'être à l'être. J'ai l'impression de retourner à l'essentiel avec ces personnes là ».

Les facteurs de motivation exprimés par les membres de notre échantillon correspondent à ceux décrits par les bénévoles en milieu hospitalier ou en maison de soins palliatifs lors d'études antérieures.

\section{Présentation des résultats}

Plusieurs thèmes sont ressortis de notre analyse. Nous allons présenter ici ceux qui ont trait de manière plus spécifique à la communication. Les actes de communication répertoriés dans cette relation d'accompagnement sont les suivants: construire la relation, poser des gestes qui parlent, parler de ce qui est significatif pour l'autre, être à l'écoute, être présente.

\section{La construction de la relation}

27 La relation entre la bénévole et le malade est une relation très particulière. Il s'agit d'une relation d'aide qui, contrairement aux autres prestations de soins (soins médicaux, nursing, psychothérapie), est non rémunérée. Bien qu'ayant reçu une formation, les bénévoles ne sont tout de même pas des expertes en thérapie. Elles sont pourtant confrontées à la souffrance, la perte et l'angoisse du malade. La relation qu'elles ont avec le patient est parfois d'« une grande intimité ». Néanmoins, elle est limitée à quelques heures par semaine. Pour Becker (1992:240) cette relation d'aide, contrairement à la relation quotidienne des proches avec le malade, est, du fait de cette brièveté, " dégagée de l'obligation de mutualité et de responsabilité réciproque ».

La position de la bénévole est donc intermédiaire entre celle du professionnel et celle $\mathrm{du}$ proche du patient. Elle ne possède ni le savoir expert, ni la connaissance intime du patient. Cela peut sembler une position désavantageuse. Cependant, ce non savoir permet d'aborder la relation sans préjugé. La bénévole va à la découverte d'un sujet vivant et non au secours d'un corps malade et d'un esprit en peine. Il s'agit tout simplement d'une rencontre entre humains. Ce qui est apporté, c'est du temps et un espace potentiel de partage. Plus que le savoir, ce qui importe alors, comme le souligne une répondante, c'est le respect :

"Un point qui pour moi est essentiel c'est le respect...quelle que soit la personne avec laquelle tu vas faire affaire, c'est de la respecter dans ce qu'elle est et dans ce qu'elle vit....Elle vit quelque 
chose de bien important. Il faut la respecter là-dedans. Si tu es respectueux des gens, tu vas tenir compte de leurs besoins. Tu vas tenir compte de leurs lacunes. Si tu es respectueux des gens ...ils le ressentent...t'as pas besoin de parler...quand c'est intérieur...ça passe par tous les pores de ta peau... ".

La construction de cette relation est un processus progressif, car il faut développer une relation qui permet d'entrer dans l'intimité des gens et cela prend du temps » dit une bénévole. Ce processus se passe plus ou moins bien selon les affinités des partenaires, comme le mentionne une répondante : «Les affinités...c'est pas quelque chose qui se commande. C'est pas parce qu'une personne est malade et qu'elle a des comportements que tu n'aimes pas que tu vas les trouver agréables ». Une autre dit : "Il faut tisser un lien..., cela peut prendre un certain temps...il faut prendre les gens où il sont...les gens malades... parfois....c'est brusques...cela peut-être impatients... cela nous fait faire une travail sur nous ".

On peut croire, qu'en principe, c'est à travers la communication que peut se créer ce lien. Cependant il arrive, au contraire, que c'est la communication même qui fait obstacle entre les interlocuteurs. Les différences de langue et de culture, de niveaux de langage, de vocabulaires, de rythmes d'élocution, etc. peuvent créer un fossé entre les partenaires. Bien que ceux-ci puissent "parvenir à une très grande intimité », parfois le courant n'arrive pas à passer. C'est pourquoi il existe toujours la possibilité pour les partenaires de remettre en question leur jumelage.

\section{Poser des gestes qui parlent}

Pour Marie de Hennezel (2001: 24), la compassion implique « d'être avec » mais dans " une façon d'être qui à un sens pour l'autre » et alors «ce qui est important c'est de faire ensemble ce qu'on...propose » c'est-à-dire qu'il faut aller dans le sens des désirs du malade.

Une de nos répondantes illustre bien cette démarche de faire avec « il y a un monsieur qui collectionnait des feuilles de musique toute sa vie...lui sa hantise c'était que cela soit jeté parce que cela n'était pas classé...le bénévole a entrepris de faire le classement avec lui, le monsieur était heureux : il communiquait son goût musical».

Une autre bénévole nous parle de sa manière à elle d'agir avec sa patiente :

«C'est ma façon à moi d'intervenir. Il y en a qui sont en meilleur état, même à deux semaines de leur décès, on fait de petites sorties. Il y a une dame, je l'amenais faire son marché en chaise roulante. Elle faisait encore partie du monde des vivants. Elle était dans le magasin, c'est elle qui choisissait ce qu'elle voulait. Faire son marché, c'est une forme de communication. J'ai appris plus de cette dame là en l'aidant à faire son marché qu'en ayant un grand discours philosophique assise à côté d'elle, parce que c'est dans la vie de tous les jours que tu connais les gens...elle va te parler de ses enfants de ce qu'elle leur faisait à manger...de fil en aiguille, c'est que des choses simples, des confidences....et puis finalement ils vont lâcher le morceau...la chose qui les stresse...quand tu sens ta mort là...les gens voudraient réparer leur mauvais coups...essayer que les gens, les proches, gardent un bon souvenir d'eux. Mais il y en a d'autres qui ne se confieront pas, qui vont se refermer sur euxmêmes, qui sont révoltés et qui vont mourir révoltés ».

En fait, chaque relation d'accompagnement est unique et se développe selon les désirs et les besoins du malade auxquels l'accompagnateur doit être attentif. Cela requiert donc une disponibilité, une ouverture, une tolérance et une grande adaptabilité. Les accompagnatrices de notre échantillon ont spontanément compris, comme de Hennezel 
(2001), l'importance de faire avec les patients les choses qui comptent pour eux et de se laisser guider par eux. Parfois ce souci de l'autre peut se manifester par de petits cadeaux :

«Une journée j'étais en train de magasiner...il y avait de belles petites crèches...je lui ai apporté ça comme cadeau de Noël...j'ai partagé beaucoup de choses avec cette personne là...avec des gestes...avec des attentions ».

\section{Parler de ce qui est significatif pour l'autre}

\section{Une bénévole raconte :}

« Il y a beaucoup de façon d'entrer en communication avec les gens...celui qui initie la communication, il faut qu'il aille chercher des points qui intéressent l'autre....y en a qui sont en train de mourir et qui ne me parlent que de ça et il y en a d'autres, c'est le contraire ils se disent «mon dieu il me reste juste tant de temps faut aussi bien en profiter...une autre qualité de la communication c'est l'ouverture...il y a une dame avec qui j'ai développé une complicité...on discutait de toutes sortes de choses...elle était anglophone (fédéraliste) et moi j'étais plutôt péquiste (séparatiste)....on avait du fun à discuter (politique) comme ça. Cela dédramatisait son état de santé. Elle a survécu 16 mois. S'il avait fallu que l'on parle juste de sa maladie 16 mois de temps, cela aurait été pénible hein ?».

La parole ainsi échangée devient relation ludique. Elle permet de vivre le présent dans la légèreté. La conscience de l'inéluctable est mise à distance momentanément. L'énergie affective du patient est ainsi renouvelée par cette conversation plus détendue.

\section{Être à l'écoute}

41 Toutes les bénévoles rencontrées soulignent l'importance de l'écoute. Pour elles, une grande partie de l'accompagnement c'est " être à côté et être à l'écoute ». C'est être le réceptacle des souvenirs, être cet autre qui permet le cheminement et l'expression de la pensée. Une accompagnatrice rapporte :

«Elle me parlait de ce qu'avait été sa vie...c'est toutes des petites confidences...je connais probablement beaucoup de choses de sa vie...une journée, en cherchant une 
photo... elle a décidé de faire le tour de ses tiroirs avec moi...Je pense qu'elle aurait eu du mal à faire cela toute seule. Elle aurait eu peur que cela la brasse trop, mais là, elle faisait ça comme pour me montrer ses souvenirs, alors c'était correct. Elle m'a montré toute sorte de choses...des souvenirs... plein de choses. En revenant, je me disais, aujourd'hui ma visite, elle a fait une différence...Je lui avais permis de manipuler tout ce qu'il y avait dans ses tiroirs, manipuler ses souvenirs émotifs, puisqu'étant donné qu'elle partageait ça avec moi...ça lui enlevait de la lourdeur... elle se disait que c'était probablement une des dernières fois qu'elle faisait ça ».

Cela « suppose une capacité d'accueil, une réceptivité non jugeante et non directrice, certes, mais également une aptitude à contenir, à être le dépositaire des affects, des émois de l'autre.» Pour Kübler-Ross, cette pionnière dans le soin aux personnes en phase terminale «cette passivité active repose sur un double mouvement d'identification et de dés-identification, de proximité et de distanciation il n'est pas donné à tous de pouvoir remplir ce mandat avec chaleur et respect envers l'autre sans s'y perdre, sans confondre leur histoire avec celle de l'autre...». Elle ajoute qu'« Il s'agit moins de comprendre, dans son acceptation intellectuelle, que d'accepter son ignorance et son incompréhension pour frayer la voie à ce qui souhaite se dire. " Parfois ce qui souhaite se dire, comme elle l'indique si bien, ce sont des sentiments négatifs, des colères, de la tristesse qu'il faut apprendre à écouter et à accepter. Selon cette psychanalyste, ce sont des réactions normales à la perte de l'identité professionnelle, de la féminité, de l'incapacité à s'occuper de sa famille et de ses enfants, de la honte d'un corps délabré par la maladie (1969/1975, p. 96). D'après ce chercheur, la dépression du patient tend à disparaitre quand il peut exprimer et assumer les préoccupations qui sont vitales pour lui. En somme, cela correspond à cette attitude d'accueil de tout ce qui peut survenir et à cette écoute qui aide le malade à se décharger de ses souvenirs qui a été décrite par plusieurs de nos répondantes.

\section{Être présente}

Tel que mentionné précédemment, le mot accompagnement signifie partage. D'après Peterson (1993 : 75) «la présence est le don de soi dans l'interaction humaine. Être présent signifie être ouvert et accessible, être à la disposition de l'autre complètement ». Pour ce chercheur, la présence est un processus dynamique. Il y a diverses formes de présence dont l'intensité va en croissant: la présence physique, la présence respectueuse à l'autre, l'attention à ses besoins, et enfin, l'intérêt pour le patient comme personne unique (Osterman et Schwartz-Barcott, 1996). Il s'agit alors de « se mettre à la disposition » du malade d'apporter au patient « une présence qui le rassure $\gg$.

Ce point de vue est largement repris par nos répondantes.

6 D'après elles, le partage peut s'exprimer par différents degrés d'intensité dans la présence. Une répondante dit: il faut "partager ce qu'il y a à partager ". Il s'agit "de recevoir ce que la personne veut partager ou de passer du temps avec elle dans un partage. Si c'est une personne qui a juste besoin d'une présence, d'une compagnie, mais qui ne veut pas partager, cela peut être aussi ça». Une autre mentionne qu'il suffit parfois de lui faire savoir que l'on est là afin d'éloigner la crainte de mourir seul. Une troisième rapporte qu'il arrive que la personne ne veuille pas être accompagnée. Il suffit de «demeurer dans

Communication et organisation, 29 | 2006 
une pièce à côté pour assurer une présence et permettre à un aidant naturel de faire autre chose ». C'est alors tout ce qui est attendu de la bénévole.

En phase terminale, l'épuisement, la désintégration corporelle, peuvent entraîner l'abandon progressif du langage articulé au profit de l'échange non verbal, du langage corporel (Giroux, 2000). Cela ne veut pas dire que le patient ne peut recevoir de communications. L'acuité auditive des patients est fréquemment mentionnée dans les études. Il semblerait même que l'ouïe serait le sens qui perdure le plus longtemps. Les observateurs des derniers moments ont aussi témoigné d'une grande sensibilité au toucher et de l'importance accordée au regard. On passerait donc, comme le suggère de Hennezel (1990), à un autre registre de la communication.

Nos répondantes nous ont décrit deux formes de présence silencieuse : la présence interactive et la présence passive.

\section{Présence interactive}

La présence peut être silencieuse et agissante. Le contact non verbal y devient alors très révélateur comme le relate une répondante :

«Le dernier Noël, quand on a défait les décorations de Noël...elle a pas parlé beaucoup...je sentais qu'elle se disait c'est probablement la dernière fois...ça se passait en silence mais on était très très proche...avec les gens malades...il y a tout l'aspect non verbal de la communication qui est très important...tout se dit pas... mais tout se ressent...il y a des choses qui se disent pas mais qui ont pas besoin d'être dites...cette journée là...il y avait comme une lourdeur dans l'air.....elle avait un côté petite fille...elle aimait décorer pour Noël...elle avait gardé une fascination pour Noël...elle était très croyante...très pratiquante...Je lui ai dit, si ça vous tente, on peut en faire des décorations de Noël. Elle n'en aurait pas faite cette année-là. Elle n'en avait pas la force ... ».

\section{Présence passive}

À d'autres moments, il s'agit d'une veille silencieuse. Une bénévole raconte qu'à son premier accompagnement :

"Je suis tombée dans une famille italienne et la dame ne parlait pas un mot de français....une dame âgée...et la dame ne savait pas qu'elle était malade, alors cela faisait une situation compliquée pour moi.....je me suis assise et, pendant des mois, je suis restée, six heures par semaine, assise sur une chaise à ne rien dire et à ne rien faire....elle pensait que c'était ma job. Il y avait toute une démystification à faire qui s'est faite avec le temps...ces gens là...le contact se fait sur un temps infini. «Cette madame là, à la fin ...j'en ai la chair de poule quand je la revois, je rentrais là, elle avait le sourire jusqu'aux oreilles. Pis, il y a des fois là, croyez le ou non, elle me parlait en italien, je lui parlais en français, et on se comprenait....elle avait fini par s'habituer à ma présence, je pense qu'elle aimait ma présence ».

51 Cette multiplicité des formes de présence va de pair, dans la littérature, avec les modalités du silence. En fait, on y retrouve décrites plusieurs formes de silence allant du silence manifestant un refus de contact au silence qui, au contraire, témoigne d'une grande intimité ${ }^{14}$. Demeurer ensemble en silence procurerait un fort sentiment à la fois de plénitude et de proximité surtout lorsque le rythme de la respiration des personnes en co-présence est synchronisé (Boerstler et Kornfeld 1995). 
52 Selon Johanne de Montigny (1990 : 97), ce que le malade désire bien souvent c'est une "présence silencieuse, une présence qui symbolise la sécurité, l'accompagnement, la vie, la continuité ». C'est peut-être la continuité et la chaleur de cette présence humaine qui était si appréciée par cette patiente italienne.

\section{Discussion}

Les quelques extraits d'entrevues présentés nous donnent un aperçu de la relation de communication entre les bénévoles et les malades en soins palliatifs à domicile, du moins de la communication telle que vécue par les accompagnatrices.

En reprenant nos trois éléments de définitions de l'urgence (temporalité limitée, enjeu important, nécessité d'agir), on apprend que dans cette situation d'urgence existentielle :

- Le temps qui est compté peut varier de quelques jours à plusieurs mois, que les bénévoles doivent « s'adapter à la temporalité » du patient, puisque c'est lui qui « donne le tempo »; au fur et à mesure que la maladie avance, la fatigue aidant, on peut s'attendre à un ralentissement moteur, à un passage de la communication verbale à la communication non verbale ;

- L'enjeu central est la signification donnée à sa vie par le patient et le souvenir qui en restera pour ses proches. Cette signification est construite à partir des souvenirs, des regrets, des remords, mais aussi des expériences vivantes des derniers moments. Le bénévole joue un rôle important dans ce processus de création de sens comme témoin, dépositaire, partenaire actif ;

- L'intervention n'est ni mouvementée, ni spectaculaire. Elle est faite le plus souvent de petits gestes et d'attentions, parfois même de silences et d'immobilité. Elle n'en produit pas moins des moments de communication intense et profonde qui motivent les partenaires à poursuivre leur relation et en particulier les bénévoles à continuer leur engagement pendant parfois plusieurs années.

Cette figure de l'urgence marque un fort contraste avec les deux autres types mentionnés précédemment. C'est sans doute parce que l'on associe le plus souvent l'urgence avec la rapidité : dans l'urgence d'un événement catastrophique, il faut agir rapidement pour sauver des vies; dans l'urgence artificielle, on stimule une augmentation de la cadence de la production pour accroître le rendement. À l'accélération de ces urgences, correspond au contraire le ralentissement progressif de l'urgence existentielle de la fin de vie.

Cependant, l'urgence existentielle possède un point commun avec ces autres figures de l'urgence: l'intensité perçue des expériences vécues. La personne en situation d'urgence expérimente soit la pression du travail intensif, la peur du danger imminent, ou la densité des moments partagés. Tous ces cas de figure ont trait à la vie (et ont donc à voir avec la mort comme le disait de M'Uzan): soit que l'on cherche à vivre au maximum, à survivre ou encore à trouver le sens de sa vie. Se questionner sur l'urgence, c'est donc réfléchir sur le lien entre ses pratiques, ses valeurs et la valeur attribuée à sa vie.

Quant à la communication, les différents thèmes relevés dans les entrevues avec les bénévoles se rapprochent de ceux qui sont décrits dans la littérature. Ainsi, une étude ethnographique américaine réalisée avec des bénévoles dans une maison de soins 
palliatifs (Foster, 2002) a révélé que les accompagnateurs considèrent les malades comme des «personnes vivantes » méritant leur écoute attentive et leur présence. Des témoignages d'accompagnateurs (médecins, infirmières et bénévoles) français confirment l'importance de l'écoute "de ses sens, de son esprit, de son cœur " (Kohler, 1995 : 29). Ils décrivent eux aussi l'expérience de la co-présence silencieuse (Daguenet, 1995).

58 Par ailleurs, les récits de nos répondantes nous ont intriguées. Ils mettaient en scène des malades d'une vitalité surprenante. Ces patients et patientes semblaient étonnamment actifs pour de grands malades, tous occupés qu'ils étaient à faire leur rangement, leurs courses, leur ballade, leur visite à l'hôpital. Nous avons découvert, grâce à ces récits, le contexte particulier de la phase pré-terminale de longues maladies, comme le cancer, vécues dans le cadre du domicile du patient. Ce contexte semble offrir une occasion très particulière de rencontre et d'échange. La relation de communication s'y déroule sur le territoire même du patient, à son heure, suivant ses règles, ses habitudes, et non dans l'univers plus contraignant et standardisé du milieu hospitalier. Les activités qui s'y déroulent peuvent suivre le rythme de ses capacités physiques, alternant mouvement et repos, paroles et silences, selon ses désirs et ses possibilités du moment. Dans ce cadre, l'expérience de communication entre le patient et l'accompagnatrice est davantage étalée dans le temps. Elle s'avère aussi très flexible. Il est donc possible de prendre davantage le temps de connaître le patient dans son propre univers, parfois entouré de ses proches. Lorsque le jumelage est réussi et qu'un lien de confiance est établi, la relation peut devenir une occasion d'être, de faire et de dialoguer ensemble.

Notre objectif de départ était de mieux comprendre le vécu communicationnel des bénévoles en soins palliatifs à domicile. La communication compatissante était définie par Kanov et al. (2004) comme une réponse empathique à la situation du malade. Les témoignages de nos répondantes ont mis en lumière un échange davantage réciproque. Il s'agit d'une communication « de l'être à l'être » ainsi que le disait une répondante.

60 L'urgence existentielle s'avère ainsi le lieu privilégié de ce que Mirashi (1999), citant Jaspers ${ }^{15}$, appelle la "communication existentielle ». Dans cette forme de communication, «le moi est avec l'autre dans un rapport de création réciproque ». Comme le dit si bien cet auteur (1999:140):

«Par l'entrée dans la relation et dans l'intériorité, chacun crée l'autre en ce sens que chacun donne à l'autre la possibilité de passer du stade de simple moi empirique, pragmatique et fonctionnel, au stade de l'existence dense et significative d'un sujet libre ».

61 Les moments de fin de vie favorisent cette communication «libératrice " Selon Marie de Hennezel , (2005: 184), il s'agit de la liberté «d'adhérer à soi». Rapportant les paroles de Marcel Legault, elle explique :

« Il y a une liberté dans l'homme qui va mourir qui n'existe pas dans l'homme qui est encore en pleine vitalité. Il y a une liberté dans le détachement, dans le dépouillement, qui permet dans une certaine mesure de dire des choses ou d'entendre des choses qui n'ont rien à voir avec ce qu'on aurait été capable de dire ou ce qu'on aurait été capable d'entendre en temps ordinaire »

62 Pour Mirashi, cette communication existentielle est fondée sur la disponibilité, la réciprocité et la responsabilité. La disponibilité suppose une attitude particulière face au temps. Il s'agit d'une générosité dans le temps consacré à la relation. Il s'agit surtout d'une disponibilité en forme d'ouverture à l'autre, lui permettant ainsi de mieux 
s'exprimer ; ouverture aussi «à la possibilité d'un nouvel être en l'autre et en soi-même » (1999 : 147). Dans ces derniers moments, quand il n'y a plus rien à perdre, on est libre d'être totalement soi. Cette ouverture se fait dans le respect et la discrétion. Les propos de Mirashi rejoignent ici ceux de nos répondantes. Celles-ci ont mentionné la profondeur de leur engagement qui va souvent au-delà des quelques heures demandées. Elles ont souligné la nature très personnelle de leurs conversations qui rend les paroles échangées confidentielles et les émotions ressenties indicibles. Elles ont évoqué l'effet transformateur de leur expérience commune.

La réciprocité suppose que chaque personne s'est engagée dans la relation en toute liberté. Elle implique l'acceptation de la liberté de l'autre, la reconnaissance de la légitimité de ses motivations et de ses raisons d'agir ou de non agir, de communiquer ou de non communiquer, comme l'ont affirmé les accompagnatrices de notre échantillon. Cela implique bien sûr, comme l'ont mentionné les bénévoles rencontrées, une grande vigilance et une attention aux désirs et aux besoins de l'autre, ainsi qu'un respect de sa spécificité. Ici Mirashi, emprunte à la phénoménologie existentielle de la réciprocité de Buber, pour qui la rencontre Je-Tu, dans le dialogue de la parole vivante, est l'expérience vécue et immédiate de la présence de l'autre. Mirashi définit alors la réciprocité de la manière suivante $(1999: 143)$ :

«La réciprocité...est la rencontre de deux consciences, opérée sans moyens ni médiations, qu'il s'agisse de la médiation par le savoir, par le travail et l'action ou par la matière. Mais cette action est un acte, c'est-à-dire une relation effectuée simultanément par chacune des deux consciences dans le mouvement de se tourner vers l'autre et de le saisir comme sujet. Cette rencontre sans médiation se fait par une sorte de "grâce», elle implique et elle est elle-même une sorte de grâce, puisqu'elle n'est en rien le résultat d'un processus ou d'une fabrication...L'autre s'y présente en effet lui-même comme conscience et liberté, et non pas « lui» comme fonction sociale ou résultat de l'histoire ».

Dans cette rencontre marquée par la réciprocité, Buber voit la confirmation, la reconnaissance et la création mutuelle des sujets. Les participants sont donc, dans cette relation, responsables de l'autre puisque, comme l'affirme Mirashi (1999: 143) :

"C'est de l'attitude de cette conscience que dépend le fait que l'autre soit réduit à l'état d'objet connaissable, exploitable ou élevé au statut de personne libre et de sujet. La responsabilité est précisément cette éthique qui décide librement d'entrer dans une relation Je-Tu afin de s'élever elle-même et d'élever l'autre au stade de sujet véritable, capable de se relier au monde et d'y déployer sa joie ».

Ainsi, la communication compatissante, comme mouvement vers autrui et réponse à sa souffrance, peut mener finalement à la communication existentielle qui constitue les partenaires en sujets libres et responsables.

\section{Conclusion}

Dans ce texte, après avoir défini l'urgence artificielle, événementielle et existentielle, il nous est apparu que l'importance de l'enjeu, la limite temporelle et la nécessité d'intervention, étaient les caractéristiques essentielles d'une situation d'urgence. Nous avons alors tenté d'explorer l'expérience de communication des bénévoles engagées dans l'accompagnement à domicile de malades souffrant de cancer. Les bénévoles rencontrées nous ont révélé que dans la situation d'urgence existentielle, les actes de communication les plus importants pour elles étaient de : construire la relation, poser 
des gestes qui parlent, parler de ce qui est significatif pour l'autre, être à l'écoute, être présente. Nous avons vu dans la discussion que cette démarche relationnelle pouvait mener à une expérience de "communication existentielle " source de liberté et de maturation pour les sujets. Il est apparu aussi que ces activités ne sont pas le propre de nos seules répondantes, puisque l'on retrouve des témoignages similaires dans la littérature sur les soins palliatifs dans d'autres contextes.

Dans l'avenir, il pourrait être pertinent de poursuivre la recherche en faisant l'inventaire d'autres situations d'urgence existentielle où cette démarche de communication pourrait être expérimentée. Nous pensons ici aux situations de chocs qui mettent à l'épreuve la capacité de résilience des sujets. Les autres situations d'urgence mentionnées dans ce texte pourraient bien offrir des cas d'urgence existentielle nécessitant un accompagnement. Il s'agit de situations où le temps s'accélère et vient bouleverser la vie des personnes, les met en situation d'échec ou de perte, ou à tout le moins, de remise en question d'un aspect important de leur identité physique, sociale ou psychologique.

Dans les organisations, cela pourrait être par exemple (Giroux, 1998) les situations d'épuisement professionnel (burn-out) qui sont de plus en plus fréquentes dans les fusions-acquisitions; dans les circonstances de fermeture d'établissements pouvant miner la survie de petites localités, ou encore dans les cas d'accidents technologiques graves en usine qui mettent en danger la vie des employés et la survie de l'entreprise. Le savoir et l'expérience communicationnels tirés de l'accompagnement pourraient sans doute être adaptés et transposés à l'intervention dans ces situations.

Quand, dans l'intensité du moment, tout bascule, il devient nécessaire de changer de registre, d'être attentif à la qualité de l'instant, de s'ouvrir au contact des consciences. On se permet alors d'entrer dans une autre temporalité parallèle, celle du kairos. L'action spontanée des personnes y est opportune, ordonnée à l'unisson, libérée de failles ou de contractions. Ce sont ces courts «moments justes et réparateurs » dont parlait Marie de Henzel (1990:79-80) qui rendent inoubliable et si précieuse l'expérience de communication existentielle en situation d'urgence.

Il s'agirait en quelque sorte d'instaurer, dans la situation d'urgence, caractérisée le plus souvent par des états de tension, de fébrilité, d'anxiété et de fragmentation, des moments de calme qui facilitent l'action lucide, conjointe, et le développement d'une attitude réaliste et sereine face à ses éventuels résultats.

\section{BIBLIOGRAPHIE}

Appelin, G., Brobäck, G. and Berterö, C., « A comprehensive picture of palliative care at home from the people involved ", in : European journal of oncology nursing, vol. 9, n4, 2005, p. 315-324. Becker, C. S., Living \& Relating: An introduction to Phenomology, London, Sage, 1992.

Boerstler, R. W. and Kornfeld, H. S., Life to Death, Harmonizing the Transition, Rochester, Vermont, Healing Arts Press, 1995. 
Boucher, A., Travail, engagement et communication : Le travail comme expérience émotionnelle positive, Montréal, mémoire de maîtrise en sciences de la communication, Université de Montréal, 2004.

Daguenet, K. « Accompagnement à domicile par le bénévole ». Bulletin JALMAV, vol. 43, 1995, p. 36-39.

Dastur, F., Comment affronter la mo t?, Paris, Bayard, 2005.

Schaller, René, «L'accompagnement existentiel des personnes en détresse», Accompagner et soignre,. no. 50, spécial 2004, p. 72-78.

Chavelli, C., Accompagner la vie de la naissance à la mort. Paris, Éditions du Rocher, 2005.

De Galzain, C., Quand les jours sont comptés, Versaille, Editions Saint-Paul, 1997.

De Hennezel, M., Mourir les yeux ouverts, Paris, Albin Michel, 2005.

De Hennezel, M., Croître jusqu'au dernier moment, Bruxelles, Alice Édition, 2001.

De M'Uzan, M., Aux confins de l'identité, Paris, Éditions Gaillimard, 2005.

De M'Uzan, M., De l'art à la mort. Paris : Gallimard, 1977.

De Montigny, J., « Distress, stress and solidarity in palliative care », in : Omega, vol. 26, n 1, 1993, p. 5-15.

De Montigny, J. et De Hennezel, M. , L'amour ultime, Montréal, Les éditions internationales Alain Stanké, 1990.

Delvaux, C., Vivre jusqu'au bout : témoignage sur l'accompagnement de personnes en fin de vie, Beauchemin, Nauwelaerts, 1997.

Feldman-Desrousseaux, É. « Des enjeux de la parole au groupe de parole » in : Manuel de soins palliatifs Paris, Dunod, 2001, p. 449-468.

Foster, E., « Lessons We Learned : Stories of Volunteer-Patient Communication in Hospice», in : Journal of Aging and Identity, vol. 7, n 4, 2002, p. 245-256.

Giroux, N. and Marroquin, L., "L'approche narrative des organisations», in : Revue française de gestion, vol. 31, nº 159 novembre-décembre, 2005, p. 15-45.

Giroux, N., « La communication ultime, le non-verbal en soins palliatifs». Dans Actes de la Conférence du Greco, "non-verbal et organisation", 2000, 25-26 mai Bordeaux.

Giroux, N., « Vivre son deuil pour s'approprier les transformations organisationnelles. » Interactions, 2 (1), 1998. p. 154-173.

Hervieux, S., La mort réconciliée, Paris, Desclée de Brouwer, 2005.

Hirsch, E., Le devoir de non-abandon, Paris, Les Éditions du Cerf, 2004.

Kavov, J. M., Maitlis, S., Worline, M. C., Dutton, J. E., Frost, P. J. and Lilius, J. M., « Compassion in Organizations ", in : American Behavioral Scientist, vol. 47, n6, 2004, p. 808-827.

Kebers, C., Soins curatifs, soins palliatifs : leurs différences, leur complémentarité, Bruxelles, Éditions Racine, 2005.

Kohler, P., « Accompagner la vie : Parole et silence », in : Jusqu'à la Mort Accompagner la Vie, vol. décembre, $n^{\circ} 43,1995$, p. 26-35.

Kübler-Ross, É. Accueillir la mort, Monaco, Édition Du Rocher, 1998.

Kübler-Ross, É. Les derniers instants de la vie, Genève, Edition Labor et Fides, 1969. 
Misrahi, R., Qui est l'autre?, Paris, Armand Colin, 1999.

Néron, S., L'art et les voix de l'accompagnemen $t$ : À l'écoute de la souffrance et de la maladie, Montréal, Médiaspaul, 1995.

Nizet, I., « Silence et accompagnement », in : Le cahier des soins palliatifs, vol. 3, n², 2002, p. 5-20.

Osterman, P. and Schwartz-Barcott, D., « Presence : Four Ways of Being There», in : Nursing forum, vol. 31, n², 1996, p. 23-30.

Peterson, C., « Presence as a Nursing Intervention With Hospitalized Children », in : Maternal-child Nursing Journal, vol. 21, n 3, 1993, p. 75-81.

Raudonis, B. M., « Empathic Nurse-Patient Relationships in Hospice Nursing ", in : The Hospice Journal, vol. 10, n¹, 1995, p. 59-74.

Richard, M.-S., Soigner la relation en fin de vie, Paris, Dunod, 2003.

Seale, C., Constructing death: The sociology of dying and bereavement, Melbourne, Cambridge University Press, 1998.

Skott, C., « Caring Narratives and the Strategy of Presence : Narrative Communication in Nursing practice and Research », in : Nursing Science Quarterly, vol. 14, n 3, 2001, p. 249-254.

Sourkes, B., The Deepening Shade. Psychological Aspects of Life Threathening Illness. Pittsburg, University of Pittsburg Press. 1982.

\section{NOTES}

1. L'urgence n'est peut-être pas perçue ou vécue de manière aussi consciente dans les cas d'accidents fatals, de coma ou de mort subite.

2. Dastur, Françoise. (2005) Comment affronter la mort ? Paris :Bayard.

3. La majorité des sujets dans l'échantillon et dans la population étudiée étant de genre féminin, nous avons choisi d'utiliser ce genre tout au long du texte par respect pour elles. Pour une fois, malgré la règle, la pratique fera en sorte que le féminin l'emportera sur le masculin.

4. De M'Uzan, M. (1977). De l'art à la mort. Paris : Gallimard.

5. Winnicott, cité dans de Montigny J. et M. de Hennezel, L'amour ultime, Montréal : Stanké, p. 30, p. 86.

6. «La mythologie grecque connaissait, à côté de Kronos, le dieu du temps qui dévore, un certain Kairos, le dieu du moment juste, du moment opportun. Confrontés comme nous le sommes à la mort dans tout ce qu'elle peut avoir de dévorant et d'angoissant, le recours à Kairos est très précieux. Quand on a le sentiment d'avoir vécu un moment juste, c'est très réparateur.» de Montigny J. et M. de Hennezel, (1990) L'amour ultime, Montréal : Stanké, p. 79-80

7. René Schaller, L'accompagnement existentiel des personnes en détresse, Accompagner et soigner. $\mathrm{n}^{\circ} 50$ spécial 2004 Accompagner les personnes en détresse pourquoi et comment p. 72

8. Chavelli, C. (2005). Accompagner la vie de la naissance à la mort. Paris : Éditions du Rocher.

9. Seale, Clive (1998) qui a étudié la construction sociale du phénomène de la mort rapporte que Lofland a qualifié en 1978 cette attitude de mouvement du «happy death». Selon Seale, ce modèle s'est développé à partir de l'observation de patients souffrant de cancer. Cette maladie, qui touche des personnes plus jeunes, fait en sorte que ceux-ci, selon Seale, ont la capacité physique et mentale d'entrer dans le "rôle prévu dans ce scénario», ce qui ne serait pas le cas de personnes parvenues en phase terminale d'autres maladies ou encore de patients très âgés.

10. Cette approche de la "bonne mort» est fortement critiquée par ceux qui sont engagés au quotidien dans l'accompagnement. Dans l'ouvrage de Chantal Delvaux (1997 : 86), Vivre jusqu'au 
bout, témoignages sur l'accompagnement de personnes en fin de vie, le Dr. Bouckenaere, oncologue et responsable d'une unité de soins palliatifs, affirme que: «Les patients demandent avant tout d'être écoutés et entendus. Si l'on est débordé par son désir de donner au patient une mort paisible et sereine, on l'oblige à mourir comme nous le souhaitons. C'est un déni de la réalité de la mort. Vouloir «fabriquer» une belle mort, vouloir compenser ce qui est horrible en offrant au patient une présence constante et en devançant ses moindres désirs a quelque chose de volontariste et d'utopique. Certains apprécient leur solitude, d'autres veulent exprimer leur colère.»

11. Matray, C. « Charte des associations bénévoles», La lettre de la SFAP, nº 6 1998, p. 4.

12. Il arrive cependant que certaines bénévoles ayant tissé des liens particuliers avec la famille et le patient poursuivent à titre privé leurs visites jusqu'à l'hôpital.

13. Comme le souligne Marie de Hennezel «on n'écoute vraiment quelqu'un que si l'on a conscience d'être en présence d'un mystère vivant, c'est-à-dire si l'on a conscience des limites de notre compréhension....il restera à jamais un étranger, radicalement autre. Aussi, l'écouter, c'est être toutes antennes sorties, réceptifs à ce mystère. Ce que nous pouvons apporter de plus précieux, c'est la profondeur de notre présence, la finesse de notre attention à l'autre. C'est être là sans jugement, sans attente particulière. Cela peut paraître simple, et cela l'est en effet, mais l'accès à cette qualité d'écoute n'est pas si aisé qu'on l'imagine. La bonne volonté ne suffit pas, car les meilleures intentions masquent souvent un projet pour l'autre qui entrave toute écoute» dans de Montigny J. et M. de Hennezel, (1990) L'amour ultime, Montréal : Stanké, p. 91

14. Le texte de Nizet, I. "Silence et accompagnement» in Les cahiers des soins palliatifs, vol. 3 $\mathrm{n}^{\circ}$ 2, 2002, p5-20. est à cet égard tout à fait remarquable.

15. Jasper, K., Philosophie, Paris, Springer-Verlag, 1932, p. 311.

\section{RÉSUMÉS}

Ce chapitre présente des résultats tirés du projet de recherche intitulé : "Communication organisante et communication compatissante : la communication dans les organisations de soins palliatifs à domicile » financé par le fonds Canadien de Recherche en Sciences Humaines (CRSH). Il décrit, dans une perspective phénoménologique, l'expérience de communication de 10 accompagnatrices bénévoles d'une association d'aide aux personnes atteintes de cancer.

This chapter presents preliminary results from a research that is entitled « Compassionate and Organizing communication: Communication in home palliative care ». This research has received a grant from the Canadian Human Sciences Research fund (CHSR). The text describes from a phenomenologic approach the communicative experience of 10 volunteers from an association helping cancer patient.

\section{INDEX}

Mots-clés : urgence, communication existentielle, kairos, soins palliatifs 


\section{AUTEURS}

\section{NICOLE GIROUX}

Nicole Giroux est professeur agrégé en communication organisationnelle à l'Université de Montréal. Sa spécialisation est la communication du changement. Ses analyses portent sur les controverses stratégiques, l'intégration discursive des fusions et acquisitions, l'analyse rhétorique et narrative des messages de changement et la communication dans les collectifs réflexifs.

\section{ANNE BOUCHER}

Anne Boucher est consultante à Vivacom. Elle réalise des interventions dans le domaine de la qualité de la communication et du bien-être dans les organisations. Sa thèse portait sur l'engagement des employés et les émotions positives vécues dans le cadre du travail. Elle est spécialiste de l'entrevue phénoménologique et de l'analyse qualitative. 